\title{
By two: A two-dimensional mobile game model for novice developers
}

\author{
Abdallah Qusef ${ }^{1}$, Abdallah Ayasrah ${ }^{2}$, Adnan Shaout ${ }^{3}$, Muhanna Muhanna ${ }^{4}$ \\ ${ }^{1,2}$ Department of Software Engineering, Princess Sumaya University for Technology, Jordan \\ ${ }^{3}$ Department of Electrical and Computer Engineering, University of Michigan - Dearborn, USA \\ ${ }^{4}$ Department of Creative Media, Luminus Technical University, Jordan
}

\section{Article Info}

Article history:

Received Sep 2, 2018

Revised Feb 5, 2019

Accepted Feb 19, 2019

\section{Keywords:}

Android

By Two

Indie game project

iOS

Mobile games

\begin{abstract}
The objective of this research was to discuss the processes and challenges encountered in the creation of mobile games for both Android and iOS platforms, which would help the novice game developers to enter the big world of mobile game industry. We highlighted each phase of the mobile game development. These involved suggestions on efficient brainstorming of ideas, proper selection of game engine, game design, number and type of pages, creation of levels, and distribution of complexity across the game level. In addition, other factors were considered such as the creation of User Interface and User Experience (UI/UX) as well as definition of the screen object's sizes and positions that would be suitable in all mobile devices. The importance of agile cycles of alpha and beta testing was also discussed, particularly its influence on the enhancement of game features. However, a successful game is recognized based on its earnings. Hence, a guide on how to properly market the game was also included. A model game called "By Two" was used in this study to illustrate and explain the steps in detail.
\end{abstract}

Copyright $\odot 2019$ Institute of Advanced Engineering and Science. All rights reserved.

\section{Corresponding Author:}

Abdallah Qusef,

Department of Software Engineering,

Princess Sumaya University for Technology,

Khalil Saket Street, Al-Jubaiha 11941, Amman, Jordan.

Email: a.qusef@psut.edu.jo

\section{INTRODUCTION}

There is an annual increase of $10 \%$ in the number of smartphone users worldwide, which reached 2.5 billion people [1]. Since mobile applications remain to be one of the most progressive industries, together with the fast growth rate of users and applications, it has to be further developed [2]. Hence, it is indispensable that the mobile game industry has also dramatically grown over the last few years, especially with the expansion of two giant platforms: Android and iOS. The mobile game industry is a huge and crowded marketplace, which makes it difficult for indie developers to be established in these markets.

However, developers are still encouraged to create mobile games as an indie project. Since the mobile game industry is an open marketplace all over the world, a developer could enter this market with lower fees. This is in favor of the indie game developers considering that the current demand and expectation for mobile applications include high quality but within the budget apps [3]. For instance, in the Google play store, a developer is required to create an account and pay a registration fee amounting to 25 USD, which involves a one-time payment [4]. On the other hand, Apple app store requires the developers to enroll in the Apple Developer Program, and shoulder memberships fees of 99 USD per year [5].

Novice game developers usually encounter several challenges when creating mobile games. These include poor knowledge on game ideas, coding, game complexity and marketing. Therefore, with minimal knowledge in terms of mobile game development, it is better for a novice game developer to 
start with 2 Dgames rather than $3 \mathrm{D}$ for simplicity. It is also better to start with a game engine with a low learning curve. Considering the nature of game and features, it is important to ensure that the chosen game engine suites the game and the features needed to be built. Game engines also help developers to build amazing games as they have rich libraries for the most needed features such as graphics, physics, ads, audio, networking and many others. Moreover, usually, these engines are cross-platform that generate builds for different platforms especially Android and iOS. They allow game creators to develop games using known programming languages such as C\# or scripting languages such as Javascript or Lua.

There are 17 and 18 game categories in Google Play Store and Apple App Store, respectively [4], [5]. Both have puzzle, board, action, arcade, word, racing, and others types of games. Categories similar to puzzle or word are easier to start with for a novice developer, since its creation is not that difficult and the user interface is not generally complicated. This consideration led us to utilize By Two for this study, which is commonly used for puzzle and swipe categories of games.

There are several methods that can be utilized in mobile game development. As an example, Yannick Francillette et al. [6] proposed a game design approach to build context adaptive games. This approach involves the design of different game scenarios with varying gameplay followed by game engine selection and proposal of the appropriate one according to the context. In 2014, Muxin Wang and Liangfei Zhu [7] also proposed some key techniques for designing and implementing an online card game based on Android 2D Graphics. It entailed the establishment of a server platform and the analysis of main Android components. In the same year, Suphawat Chungthanacharoenlert and his colleagues [8] discussed a math game on android from different aspects like game story, system overview and design, development tools, level design, system implementation and algorithms, and finally, the user interface. These approaches will serve as guides in designing, creating, and implementing appropriate strategies.

The purpose of the game will also provide ideas on the type of game to be created. For instance, if a mobile game aims to improve the meta-cognitive skills of students, a suitable game like animated puzzles should be created. This was the inspiration of a 2018 study by Sudin and colleagues to improve the thinking ability of students [9], which presented the fact that games are need-based. On the other hand, if the goal of the developer is to create an adaptation type of game, there is a need to analyze the knowledge and game skills of the users, similar to the game idea of EQUAFUN developed by Belahbib and his coauthors in 2016 [10].

Meanwhile, Ana Vasconcelos et al. (2012) [11] conducted a study that provided the 10 rules of thumb for tablet-based games. They particularly designed and analyzed a game for seniors, wherein they successfully developed prototypes for a gaming cognitive platform, evaluated the end-users, and iteratively improved. The output of their research will be beneficial in cases wherein the mobile games are created for specific users, including children, disabled and the elderly.

In 2017, Mohamed Ali and his fellow authors [12] conducted a comparative study on the same application but published on different application stores. Through their study, they have successfully addressed some cross-platform development challenges. The comparison provided an in-depth evaluation of the possible difficulties that game developers might face in various application stores. Novice developers will be mostly benefited by this study due to their need to consider all possible challenges along the way.

Game improvement is also necessary in order to provide a better user experience. User feedback is essential in creating improved version of the mobile application. For example, a study in 2017 showed that a tourism app in Indonesia utilized user feedback to improve the latest version. Using the data gathered, Tahyudin and Saputra were able to improve user satisfaction [13].

Mobile games are not limited to entertainment purposes. It can also be used to study the behavior of the users in certain conditions. Tuang Dheandhanoo and his co-authors (2016) [14] described an analyticsbased approach that utilizes gameplay metrics to measure the effect of the marketing campaign on the user behavior on a popular mobile game. They explored the effectiveness of the marketing campaign by comparing the gameplay metrics between the pre-campaign, campaign, and post-campaign period and explained the results.

User interface and User experience is another major contributing factor in order to engage mobile game users. However, many developers fail to consider this aspect, which leads to less game interaction and interest. Hence, it is essential to know the advantage and importance of using different types of mobile game interaction [15]. In return, this will provide game ideas and possible improvement of game features. Hence, the challenge for indie game developers lies in building an amazing game from one side and knowing how to promote and market this game to the other side with the minimal knowledge on hand.

In 2006, Edwards and Coulton [16] identified the need for providing the necessary skills to facilitate innovative game development as well as encourage entrepreneurship through collaborations. Through this research, we aim to address and guide the indie game developers in creating a mobile game journey and 
attain success in the mobile game industry.

The remaining parts of this paper are organized as follows: Section 2 will present the methods involved in developing the mobile game. Second is Section 3, which will present the results the study. Next is Section 4 or the conclusion of the study. Lastly, Section 5 will identify the future applications of this research.

\section{RESEARCH METHOD}

For developing a 2D mobile game, the developer must consider the target audience, create an engaging gameplay and strategically monetize it. To prepare this process usably and traceable for the reader, we describe the development phases of a mobile game into subsequent sections; hence it can be used as guideline and aid in the creation of personal brand in the mobile gaming industry. The major steps for $2 \mathrm{D}$ mobile game development are presented in Figure 1.

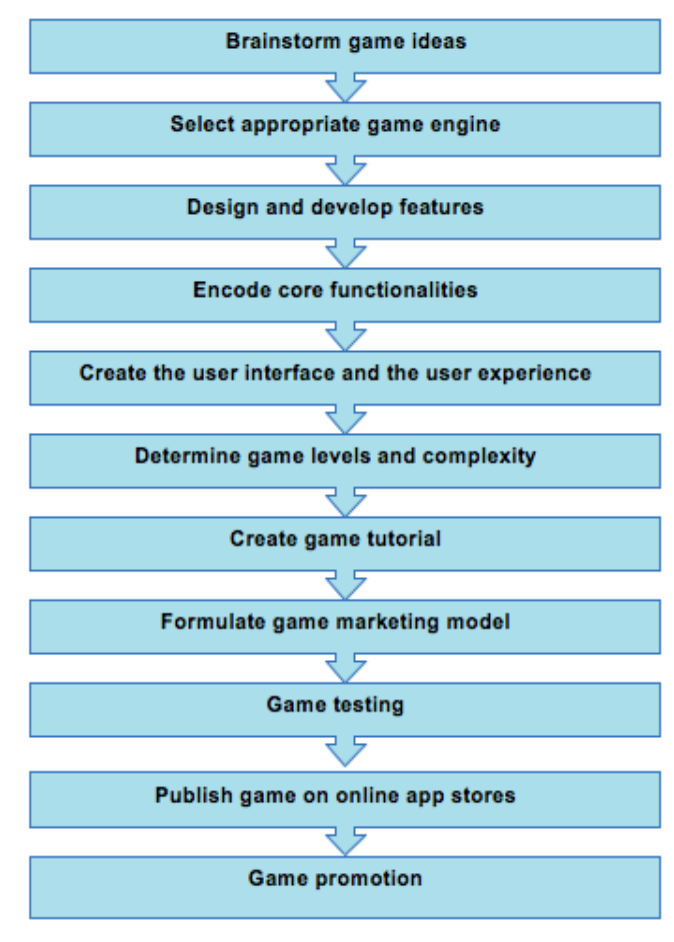

Figure 1. Key steps in creating a 2D mobile game

\subsection{Building The Idea}

The idea of the game plays a key role in its success. Genuine and sharp ideas have more chances to be successful than just copied ideas. One of the ways helps in brainstorming ideas is to explore games on app stores and try to derive new ideas from them. The derived idea should be distant from the copied idea. For example, for puzzle games, there is a wide space for innovation. This can be done by exploring many existing puzzle games on stores and math puzzles, as well as the possibility of converting it to mobile puzzles. Once the developer gets a new idea, it is advisable to sketch the idea on a paper. This would highly help in exploring and building the idea in a more realistic way.

\subsection{Choosing The Game Engine}

Currently, there are many game engines available. However, selecting the wrong game engine can lead to a closed road and financial losses because the developed product may not live up to the standards due to lack of features in the selected engine [17]. Choosing the right game engine depends on several factors, such as developer experience in game development, game engine learning curve, free or paid, one or crossplatform, engine performance, community support, documentation and resources, and 2D and/or 3D support. Some of the top existing game engines are as follows: Unity, Cocos2d, GameMaker, LVE, and Corona SDK. In this study, the Corona SDK was chosen based on the following factors: 
a) It is a cross-platform that generates APK build for android as well as APP build for iOS. Thus, a developer can write the code once but can build many platforms.

b) Coding simplicity is one of the key factors for choosing this engine. There is no complicated IDE screen with full of buttons. It only involves plug and play in which the developer can enhance and learn his/her development skills incrementally in the duration of the project.

c) Corona is built on top of Lua Language [18] and rich in Plugins, libraries, and APIs for all the developer's needs. Some of these libraries include physics, graphics, network, database and APIs, including integration with advertising providers and many others.

d) On the other hand, the Corona simulator is a powerful, fast, user-friendly, and allows many device view options for iPhone, iPad, Samsung, HTC, Kindle and even for customized mode. Hot deployment is a plus, once the developer saves the new changes, the simulator directly reflects these changes on the simulated device.

e) Lastly, it entails no cost for it is a totally free engine.

\subsection{Design \& Development}

In this phase, a developer needs to do numerous paper sketches to clarify their idea. The number and type of pages are considered. One important factor that supports these steps for a beginner and an indie developer in the gaming industry is the installment of a group of mobile games with the goal of obtaining ideas and features, the number of pages, the navigation process, and the available features.

\subsection{Building Core Functionalities}

Commonly, it is advisable for a novice developer to start implementing the core functionality of the game. In other words, there is a need to develop the play page. The developer needs to create the main algorithms for the game and make sure that it is a double game to be able to prove the concept.

\subsection{Building the UI and the UX}

The creation of the UI and the UX is one of the most important parts of game development. A developer has to decide what pages will be placed inside the game. In this study, several pages were placed: menu page, packages page, levels page, play page, market page, how to play page, and about the pages are selected based on the Flow Free approach [19].

An attractive and simple design would highly lead to the game's success. A developer may not have the ability to create all the needed icons inside the game, so the help of a designer is needed for this purpose. As these mobile games will be deployed on many and different devices with different screen resolutions and sizes, screen objects must be adaptive. Dynamic screen object sizes and positions could be ensured using equations that define them by screen width and height.

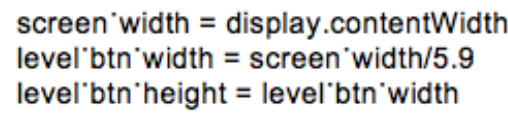

Figure 2. Dynamic defining level icon size

Figure 2 shows how to define screen objects dynamically based on the screen dimensions. The first line refers to the screen width of the device using the display library provided by Corona. Then, after some trial and error, we found out that the best level icon width is equal to the screen width divided by 5.9.

Another aspect that must be defined dynamically is the position of screen objects. Figure 6 shows how to dynamically determine each level position on the screen. It defines the centerX variable, which places the screen center horizontally. This variable could also be determined from the display library.

To define the position of an object, two values namely $\mathrm{X}$ and $\mathrm{Y}$ are needed. In our example, a level object is defined based on centerX value and level column value (j). On the other hand, the level $\mathrm{Y}$ value could be defined based on level row value (i) with some padding as presented in the equation (Figure 3 ).

\subsection{Generating Levels And Complexity}

Level-oriented games must have different degrees of complexity. There are various approaches available to organize complexity. Some games categorize levels into easy, medium and hard packages, while others are distributing different levels of complexity within one package. 


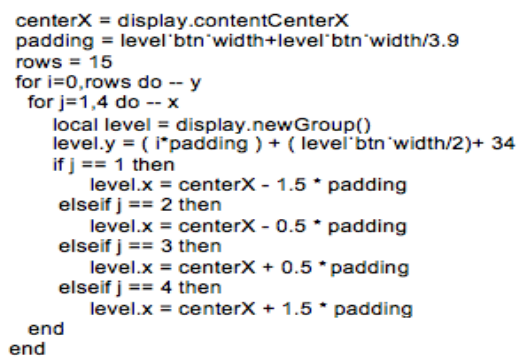

Figure 3. Dynamic defining level icons positions

\subsection{Building How to Play Tutorial}

Mobile games are published in stores for all kinds of people. The level of smartness differs from one person to another. Hence, some will get the idea of the game directly, while others will take time to understand what the game is. For these reasons, how to play is an important part of the game. Usually, game makers put a tutorial play once the player clicks on the first level to the enforce player to watch it.

\subsection{Developing the Business Model and the Market}

Indie game developers are passionate to develop games and publish them for the people to enjoy. However, earning money from developing games is also a key target. Strategies vary across types of games, wherein some are publishing paid games but others are creating free games with In-app-purchases. These purchases are extra features of the game that a player needs to pay in order to unlock special features. Another method to earn money is through advertisements inside the game. These ads may be in several forms such as the top or down banner, full-screen image and video ads, which are the most profitable ads mechanism.

\subsection{Alpha and Beta Testing}

Once the developer has completed the game, he can ask people to try the game and obtain feedback, which will help enhance the game. This could be achieved through Alpha and Beta testing for the games published in Google play store and TestFlight beta testing for games published in Apple App store.

This process should take place as agile cycles of testing, getting feedback and enhancing until the game becomes mature enough and fulfills the players wants and needs. This is a very important phase for it will absolutely provide the developer valuable information and feedback, which can enhance the playing experience or add new features to the game.

\subsection{Publishing To Google Play and Apple App Stores}

Publishing on both Google play store and Apple App store requires the developers to pass through many steps to get accepted. Between the two, Google play store is more convenient and less complicated. Acceptance and publishing of games are done within 24 hours. It also allows publishing of low-quality apps. As of January 1, 2018, the transaction fee for subscription products is $15 \%$ [20].

On the other hand, Apple App Store needs more time for acceptance and publishing, which usually takes more than three days as it passes through a review process. To generate a build, MAC device is needed for it uses Xcode to generate the App build. App Store may be more profitable since its users already have the sense of paying for soft things like apps and e-books. Apple announced that commissions for all iOS inApp purchases would be reduced from $7 \%$ to $2.5 \%$ globally [21].

Similar to a YouTube video, the users first see the screenshot of the video and read its title. If they get attracted, they will play within the first 10 seconds then either complete or leave that video. Comparing this scenario to both stores, users initially see the icon and name of the game, so it must be attractive enough to impress the users and encourage them to click on and navigate to the game page. For both stores, the developer should upload screenshots for the game, which will also support user acquisition. Google play and Apple App stores provide the option to a developer to upload an App preview video for his game and this can serve as a key for attracting users to click the download button.

\subsection{Marketing Strategies}

Once the game is published and available in both stores, a developer needs to promote his game to the audience. In fact, this is not an easy task for indie game developers as most of them are of technical background and lack the necessary marketing experience. 
The first step in marketing is to have a good game in all aspects: idea, attractive user interface, enticing player experience, nice icon, and name. However, these factors alone will not push the game to the frontline of stores directly. This is just the initial step. Marketing is a long journey and needs experience, budget, and patience.

There are many marketing strategies for promoting a mobile game such as App Store Optimization (ASO), paid campaigns on Facebook, Instagram, Twitter or any other channel that could reach the target audience. Another marketing mechanism is to localize the game to the target languages and countries such as English, Chinese, Russian, etc.

There are a number of factors that dramatically affect game promotion, including game ratings, reviews, and the number of installs (installs growth). The developer can take care of these factors in many ways by placing a button or pop-up inside the game that asks users to rate the game. Once this button is clicked, the user will be redirected to the game page of the store.

For a newly published game, installs growth, good reviews, and good rating are essential matters for promoting the game. One of the ASO techniques is to increase these three areas one way or another. Some parties provide these as paid services with different package options. For example, they offer per install, per install with the review or per keyword App install, which dramatically enhances ASO and so on.

A developer could also perform A/B testing in which the developer tries one marketing strategy at a time and determine which one gives the best result that they can focus on. Apart from the previously stated marketing strategies, game creator also has the option to work with a game publisher and throw all the marketing work to the publisher side. In this setting, the publisher will share profits with the developer.

\section{RESULTS AND ANALYSIS}

In this case study, the "By Two" board puzzle game was used as a model. This game was based on the idea of removing all the tiles on board by continuously dividing these tiles starting from the highest value.

In terms of the game design, the menu page, as shown in the left side of Figure 4, is the landing page of the game. This page contains the game logo on the top, followed by the "Play" button, the "How to Play" button, and four buttons on the bottom of the screen. These four buttons refers to About, Market, Settings and Share game buttons.

Currently, By Two offers two packages, 44 and 55, which pertains to the board size. The right part of Figure 4 shows these packages. Once the player clicked on one of these packages, the game navigates to the levels page as shown in the left side of Figure 5. Levels are locked and get unlocked sequentially. Once the player passes a level, the next level will be unlocked. The ranking is based on three stars for best, two stars for good, and one star for low ranked levels.

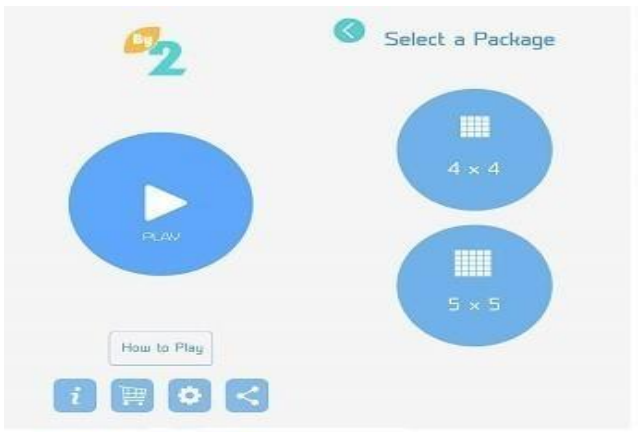

Figure 4. Menu and packages page

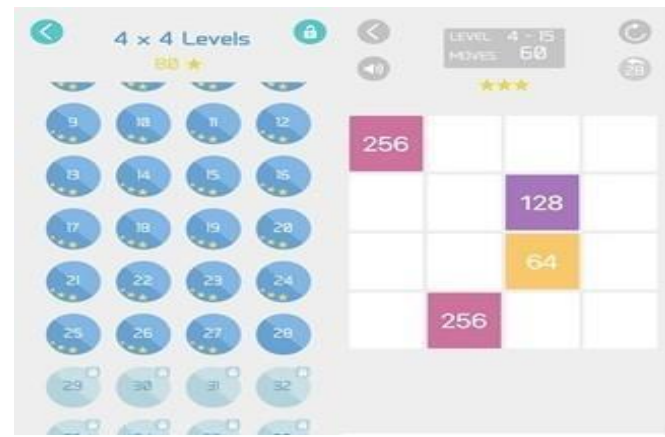

Figure 5. Levels and play page

Play page, as it appears in the right part of Figure 5, presents a board that contains several tiles with values of base 2 . The user swipes to move tiles in any direction (left, right, up and down). Once two similar tiles hit, they merge and divides by two. The player keeps on dividing tiles until the board is empty. However, the number of movements is restricted in each level to make it more challenging. The player must start dividing tiles from the maximum number on board to the minimum in order to clear all tiles and win. A player will lose if he ran out of moves or the board is blocked of tiles without any similar adjacent tiles.

Market and how to play page is shown in Figure 6. On the left side, several game features are placed. In-App purchases are added in the Market page, wherein different features with different prices are 
presented. Even the removal of ads could be sold as a feature for the users. The players may also opt to buy additional moves or undo their move. They may even pay to unlock all levels of each package. However, since not all players are willing to navigate all the pages of the game, the developers must design their games in a manner that reroute the players to the market page or to push the feature in order to unlock the offer on screen once needed. One of the common in-app purchases is the offer for the players to continue playing even after losing by watching a video advertisement. This is also a good method for the game developers to earn.

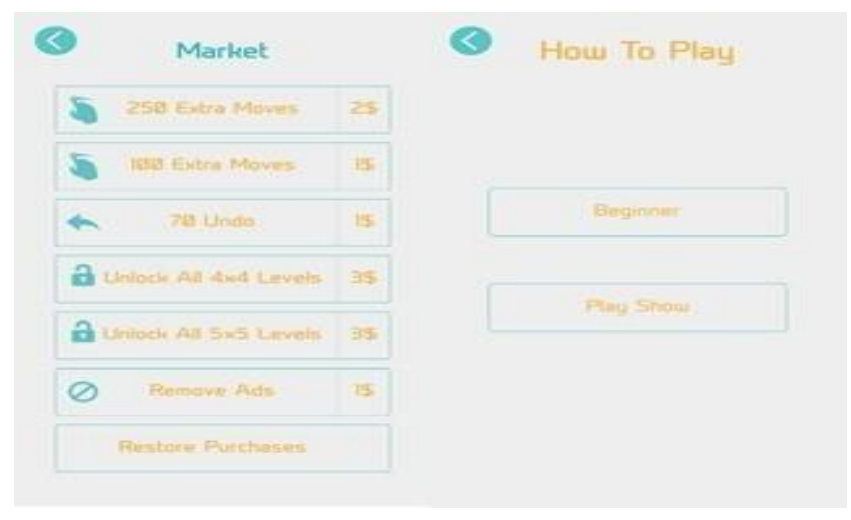

Figure 6. Market and how to play page

A game tutorial and play show is also placed on the right side of the market page. This game features will help the users be familiar with the instructions and fully understand the game. The "How to play" video presents rules and guidelines while the other one is a sample play show. To ensure that the player sees these tutorials, the basic guidelines appear on level one, but the play show appears on level twenty.

Meanwhile, the left part of Figure 7 shows the Settings page, which enables the player to change the background, turn sounds on and off, and rate the game at the store. This feature allows the game to be userfriendlier, since it adapts the user interface to the preferences of players. On the other hand, the right section of Figure 7 presents the current game version, developer name and e-mail address.

At the moment, By Two has 60 levels for each package, wherein each row has 4 levels. Therefore, the total number of rows is 15. The levels 1-20 are labeled as easy, as a way for the player to understand the game and break the ice. Levels 20-30 are of medium complexity that will allow the player to feel the challenge. Levels 30-60 are distributed between medium and hard. For example, three sequential levels come with medium complexity followed by a harder level so the player will feel rested but also excited. On the other hand, all 55 levels are between medium and hard complexity. The differences in the complexity of levels allow the players to adjust and improve their game skills through time.

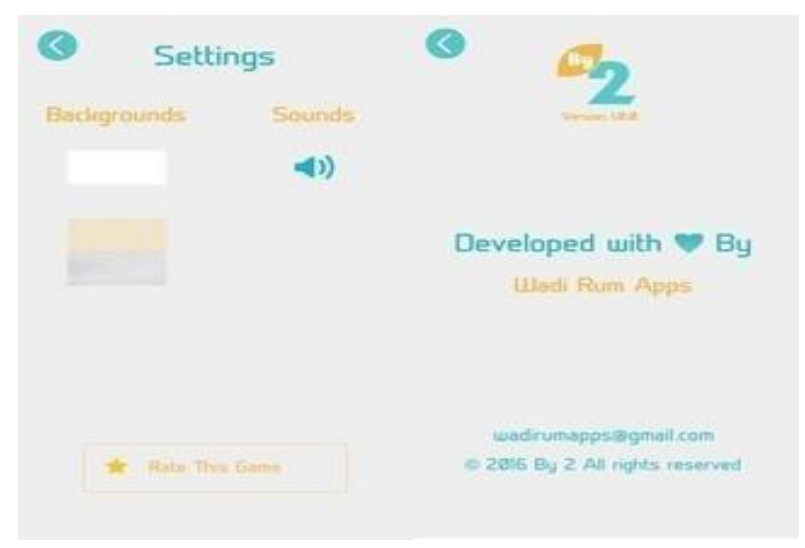

Figure 7. Setting and about pages 


\section{CONCLUSION}

In this paper, we highlighted the journey the novice game developers go through for developing a 2D mobile game for Android and iOS. The aim was to inform and guide the developers on how to start and enter the industry successfully. Under this goal, we addressed and discussed all the main stations on the road starting from choosing the game engine, developing the game idea, as well as the designing and the development of the user interface. Successful games start with the correct game idea that can capture the interest of the target audience. Hence, it is essential for new developers to consider these aspects prior to the creation of the 2D mobile game.

We also explained the importance of alpha and beta testing, feedback from users together with agile cycles of enhancements based on these feedbacks. The results of these tests and responses from the alpha and beta users allow room for further improvement. Any errors and additional features may be corrected and added, respectively. It is necessary for published games to have fewer problems if not error-free in order to attract more players. In addition, we discussed the ins and outs of publishing games in Google Play and Apple App stores, as well as how to promote the game using ASO.

The journey of novice game developers in the mobile game industry will be challenging. However, with the necessary knowledge, skills and strategies, they will obtain the highest potential number of players, ratings and income.

\section{FUTURE APPLICATION}

The results of this study will be beneficial for the new mobile game developers, who attempt to infiltrate the industry despite the large number of competitors globally. This will also serve as possible guide for related researches in the future. Since this only involves a basic guideline and information on mobile game creation, a more in-depth evaluation of the process is needed. It may also include possible approaches for building level complexity, or research on effective marketing of indie games. Another possibility is to create a 3D game guide for the novice game developers and identify the possible challenges they may encounter along the way.

\section{REFERENCES}

[1] K. Tao and P. Edmunds, "Mobile APPs and Global Markets," Theoretical Economics Letters. vol. 8, pp. $1510-1511,2018$.

[2] S. Vaupel, et al., "Model-driven development of mobile applications for Android and iOS supporting role-based app variability," Software \& Systems Modeling. vol. 17, p.35, 2018.

[3] K. Anureet and K. Kaur, "Effort Estimation in Traditional and Agile Mobile Application Development \& Testing," Indonesian Journal of Electrical Engineering and Computer Science, vol. 12, pp. 1265-1272, 2018.

[4] Google. How to use the play console - play console help. [Internet]. Google; 2018. Available from: https://support.google.com/googleplay/android developer/answer/6112435?hl=en.

[5] Apple. Choosing a membership, purchase and activation -support - apple developer. [Internet]. Apple; 2018. Available from: https://developer.apple.com/support/compare-memberships.

[6] Y Francillette, et al., "Adaptive gameplay for mobile gaming," Computational Intelligence and Games (CIG) IEEE Conference, pp. 80-87, 2017.

[7] M. Wang and L. Zhu, "Designing and implementing an online card game based on android 2d graphics," Audio, Language and Image Processing (ICALIP) International Conference, pp. 817-821, 2014.

[8] S. Chungthanacharoenlert, et al., "The golden sugarcane: A math game on android," Student Project Conference (ICT-ISPC) Third ICT International, pp. 29-32, 2014.

[9] I.S. Sudin IS, et al., "Adaptation Meta-Cognitive as an Educational Tool: Animated Puzzle," Indonesian Journal of Electrical Engineering and Computer Science, vol. 12, pp. 319-325, 2018.

[10] A. Belahbib, et al., "Serious Games Adaptation According to the Learner's Performances." International Journal of Electrical and Computer Engineering, vol. 7, pp. 451-459, 2017.

[11] I. Tahyudin and D.I.S. Saputra, "A Response Analysis of Mobile Augmented Reality Application for Tourism Objects," International Journal of Electrical and Computer Engineering, vol. 7, pp. 3500-3506, 2017.

[12] A. Vasconcelos, et al., "Designing tablet-based games for seniors: the example of cogniplay, a cognitive gaming platform," Proceedings of the 4th International Conference on Fun and Games, pp. 1-10, 2012.

[13] M. Ali, et al., "Same app, different app stores: A comparative study," Proceedings of the 4th International Conference on Mobile Software Engineering and Systems, pp. 79-90, 2017.

[14] T. Dheandhanoo, et al., "Game play analytics to measure the effect of marketing on mobile free-to-play games," Science in Information Technology (IC-SITech) 2nd International Conference, pp. 125-130, 2016.

[15] P. Chaichitwanidchakol and W. Feungchan, "Exploring Mobile Game Interactions," International Journal of Electrical and Computer Engineering, vol. 8, pp. 3954-3965, 2018.

[16] R. Edwards and P. Coulton, "Providing the skills required for innovative mobile game development using industry/academic partnerships," ITALICS, vol. 5, 2006. 
[17] Z. Ali and M. Usman, "A framework for game engine selection for gamification and serious games, " Future Technologies Conference (FTC), pp. 1199-1207, 2016.

[18] Corona. Cross-platform game/app development toolset. [Internet]. Corona; 2018. Available from: https://coronalabs.com.

[19] G. Play. Flow free-apps on google play. [Internet]. Google Play; 2018: Available from: https://play.google.com/store/apps/details?id=com.bigduckgames.flow.

[20] Google. Transaction fees-play console help. [Internet]. Google; 2018 Available from: https://support.google.com/googleplay/android-developer/answer/112622?hl=en.

[21] iTunes. Commission update, - itunes affiliate resources. [Internet]. iTunes; 2018: Available from: https://affiliate.itunes.apple.com/resources/blog/commission-update/.

\section{BIOGRAPHIES OF AUTHORS}

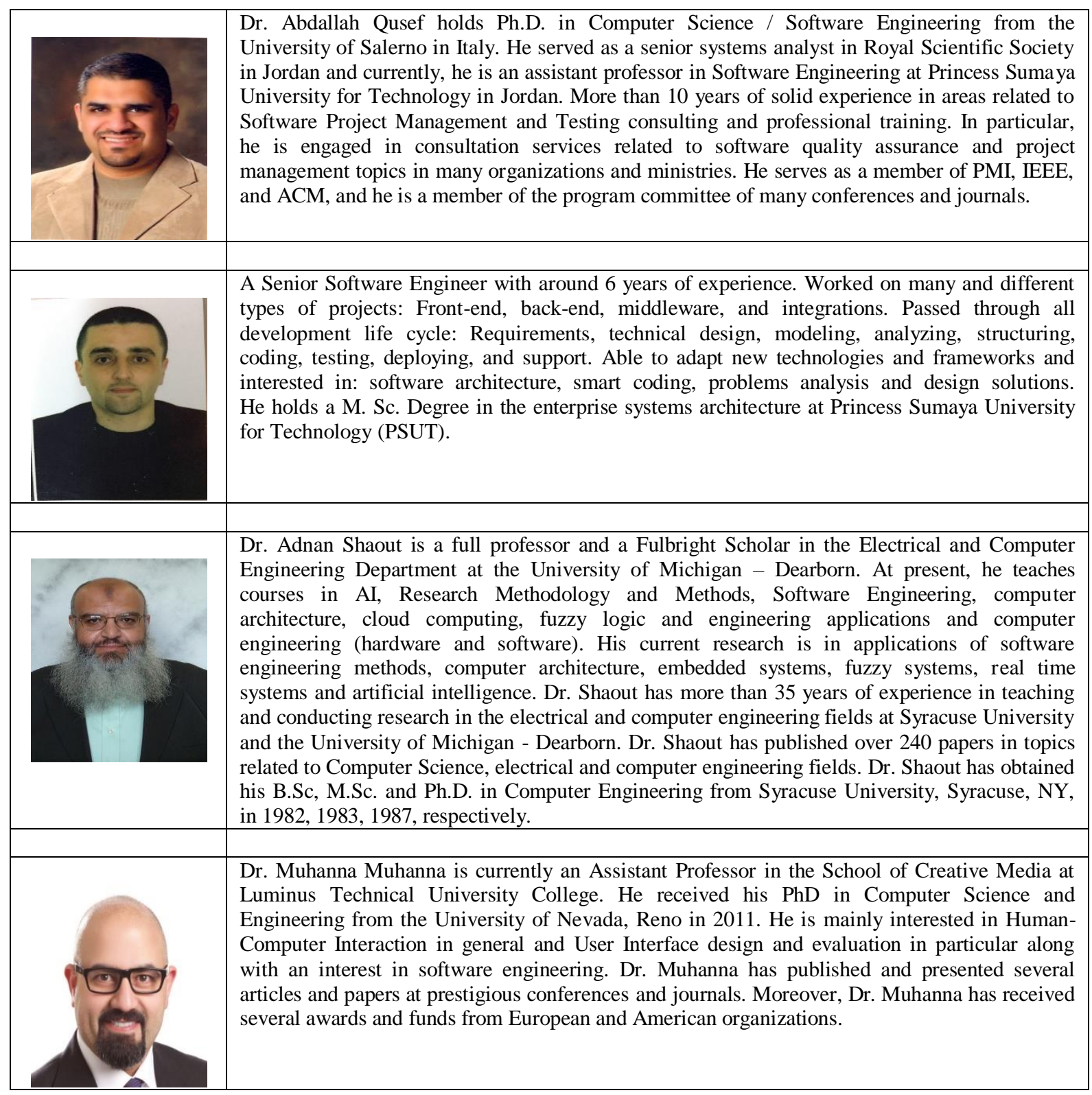

